\title{
Hard body amphiphiles at a hard wall
}

\author{
JOSEPH M. BRADER ${ }^{1 \dagger}$, CHRISTIAN VON FERBER ${ }^{2}$ and MATTHIAS SCHMIDT ${ }^{3^{*}}$ \\ ${ }^{1}$ The James Franck Institute, University of Chicago, 5640 South Ellis Avenue, \\ Chicago, Il 60637, USA \\ ${ }^{2}$ Theoretical Polymer Physics, Freiburg University, Herman-Herder-Straße 3, \\ 79104 Freiburg, Germany, \\ ${ }^{3}$ Institut für Theoretische Physik II, Heinrich-Heine-Universität Düsseldorf, \\ Universitätsstraße 1, D-40225 Düsseldorf, Germany
}

(Received 18 December 2002; revised version accepted 17 January 2003)

\begin{abstract}
We investigate the structure of amphiphilic molecules exposed to a substrate that is modelled by a hard wall. Our simple model amphiphiles consist of a hard sphere head group to which a vanishingly thin needle tail is radially attached, resulting in a lollipop shape. Such particles act as amphiphiles when added to a binary fluid of hard spheres and needles. Focusing on the pure amphiphile system we compare the results for the positional and orientational order profiles obtained from a recent density functional approximation to those of our computer simulations and find good agreement. For low densities the structure is ruled by the loss of orientational free volume near the wall, while for higher densities packing of the spherical heads dominates. Furthermore, we test the wall sum rule explicitly for this model fluid and find rich structure of the contact distribution which can be interpreted in terms of typical particle configurations.
\end{abstract}

\section{Introduction}

In order to understand the behaviour of ternary water-oil-surfactant mixtures on a microscopic level, different model systems have been utilized, ranging from very simplified phenomenological theories to more realistic simulation studies. The principal objective of such research is to start from model interactions between individual molecules and to predict macroscopic properties such as phase behaviour and structural correlations [1-6]. Often the attractive parts of the pair potentials are specifically tailored to generate the required amphiphilic properties. However, it was shown by computer simulations that hard body interactions alone are sufficient to generate mesoscopic micellar structures [7], using a model of amphiphilic molecules immersed in a hard sphere fluid. As a model for mesogens, van Duijneveldt et al. investigated the phase behaviour of hard spherocylinders with a flexible tail attached to either endcap [8] and to only one of the endcaps [9]. They found that the presence of the tails enhances the stability of the smectic-A phase and suppresses the nematic phase.

\footnotetext{
*Author for correspondence. e-mail: mschmidt@thphy.uniduesseldorf.de. Present address: Debye Institute, Utrecht University, Princetonpln 5, 3584 CC Utrecht, The Netherlands.

†Present address: Institute of Physiology, University of Bern, Buehlplatz 5, 3012 Bern, Switzerland.
}

Recently, a similar simple model that features hard body interactions only was proposed [10]. To a binary mixture of hard spheres and vanishingly thin needles [11], a third component is added that consists of a sphere and a radially joined needle. Besides being the 'natural' amphiphile for the sphere-needle mixture, this mimics the shape of the hydrophilic headgroup and hydrocarbon tail of real amphiphile molecules. The appeal of the model stems from the absence of an energy scale, hence its behaviour is governed solely by the (three) densities of the species and the ratio of needle length and sphere diameter as an additional geometric parameter that determines the amphiphile strength. In the framework of density functional theory (DFT) [12, 13] extending Rosenfeld's work [14-16] and following the treatment of a binary sphere-needle mixture [17], a geometry-based DFT for this model was proposed [10]. The bulk fluid equation of state derived from the theory was found to be in good agreement with that obtained from computer simulations. Theoretical results for the fluid demixing phase behaviour of the binary subsystems (obtained by setting the density of one of the components to zero) supported the amphiphilic character of the model. In particular it was found that compared to the demixing binodal in the sphere-needle mixture, the mixed region grows (demixing is suppressed) if either component is replaced with amphiphiles. Besides the ternary bulk phase behaviour (which has not been considered so far), the crucial test to prove the validity of the model is 
whether the interfacial properties are in accordance with those of real ternary surfactant mixtures. Clearly, this is a potentially demanding project, and as a prerequisite, in the present study we seek to understand the nature of correlations that emerge from the shape (and hence the interactions) of the model amphiphiles.

We choose a generic situation, the hard, impenetrable wall, to study the packing effects caused by the hybrid shape of the particles. Besides the intrinsic interest in amphiphiles at surfaces, our aim is also to assess the accuracy of the theory in inhomogeneous situations. In contrast to fluid-fluid interface studies, the behaviour near walls can be obtained more easily from computer simulations, and hence we have carried out Monte Carlo (MC) simulations for the pure system of amphiphiles against a hard wall.

We find that for low densities the behaviour is driven by the (trivial) loss in rotational free volume (accessible particle orientations) that arises from the overlap of the particle tail with the wall. Increasing the bulk density leads to a crossover to behaviour that is governed by the packing of the heads, and thus resembles that of the hard sphere fluid. Theoretical and simulation results agree well, given the complexity of the situation, also very close to the wall. The peculiar shape of the second peak is captured correctly but its height is slightly overestimated. Our theoretical results for the wallparticle contact distribution show rich structure, which we can relate to special geometric particle arrangements at the wall.

The paper is organized as follows. In Section 2 we define the model explicitly. Section 3 is devoted to the density functional and computer simulation methods. In Section 4 we present results and we conclude in Section 5 .

\section{Model}

We consider amphiphilic hard core particles which consist of a hard sphere of diameter $\sigma$ and a vanishingly thin needle of length $L$. The needle (tail) is attached radially to the sphere (head). We take the centre of the sphere as the position of the particle (denoted by $\mathbf{r}$ ) and denote the direction of the tail by a unit vector $\boldsymbol{\Omega}$, see figure $1(a)$. The interparticle interactions are such that both sphere-sphere and sphere-needle overlaps are forbidden. The excluded volume between two needles is zero owing to their vanishing thickness. Hence configurations with overlapping needles carry vanishing statistical weight, and the needle-needle interactions can be assumed as being ideal. The wall (see figure $1(b)$ ) is impenetrable to both the head and tail. Let $\theta$ be the angle between $\boldsymbol{\Omega}$ and the surface normal towards the wall (see again figure $1(b)$ ), and let $z$ be the component of $\mathbf{r}$ perpendicular to the wall. Then we can describe the

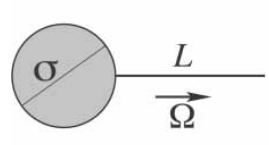

(a)

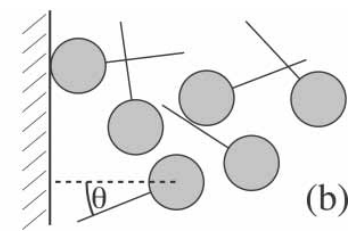

Figure 1. Sketch of the model amphiphilic system. (a) Each amphiphilic molecule consists of a hard sphere of diameter $\sigma$ to which a vanishingly thin needle of length $L$ is radially attached. The particle orientation is described by the unit vector $\boldsymbol{\Omega}$. (b) Typical configuration of amphiphiles against a hard wall. The needle tails may overlap with each other owing to their vanishing excluded volume, but they can penetrate neither the spheres nor the wall.

wall as an external potential

$$
V_{\text {wall }}(z)= \begin{cases}\infty & z<z^{+}(\theta) \\ 0 & \text { otherwise }\end{cases}
$$

where $z^{+}(\theta)$ is the distance of closest allowed approach between the sphere centre and the wall, given by

$$
z^{+}(\theta)= \begin{cases}(L+\sigma / 2) \cos \theta & \theta<\arccos [1 /(1+2 \mathrm{~L} / \sigma)] \\ \sigma / 2 & \text { otherwise }\end{cases}
$$

The system is governed by only one thermodynamic parameter, which we take to be the packing fraction of the spherical heads, $\eta_{\mathrm{A}}=\rho_{\mathrm{A}} \pi \sigma^{3} / 6$, where $\rho_{\mathrm{A}}$ is the amphiphile number density. The size ratio of needle length and sphere diameter, $L / \sigma$, is a further geometric control parameter.

\section{Methods}

\subsection{Density functional theory}

As a theoretical approach to the study of the interfacial properties of our model fluid we use the DFT of [10] which extends Rosenfeld's fundamental measures theory [14-16] to an example of non-convex particles with orientational degrees of freedom. The only inputs to the theory are a geometrical description of the particle shape and exactly known limits for situations of extreme confinement. The theory in [10] is formulated for a multicomponent mixture. Here we apply it to a one-component system (monodisperse in sphere diameter and tail length). As an essential feature, this DFT describes the many-body behaviour of the system in terms of geometrically determined weight functions that correspond to the particle shape. The weight functions are used to obtain weighted densities by building convolutions with the actual density profile. The excess (over ideal gas) free energy density is expressed as a function of these weighted densities and integration over space and orientational degrees of freedom yields the excess free energy. For the full definition of the weight functions, that of the free energy density, and all further technical details we refer the reader directly to [10]. 
The numerical minimization of the density functional in the presence of the external potential, equation (1), is done via a standard iteration technique, and follows closely that of a related investigation in a binary sphereneedle mixture [18].

\subsection{Computer simulations}

In order to obtain benchmark results, we performed canonical Monte Carlo simulations with 500 particles. Periodic boundary conditions are used in both directions parallel to the walls. The two walls have square shapes. The distance between both walls is taken to be twice their lateral length, in order to reduce finite-size effects. For the simulated total densities $\eta_{A}=0.05$, $0.10,0.15,0.20,0.25,0.30$ we performed $2.1,2.7,3.6,4.4$, 4.7, 7.0 million MC moves per particle, respectively. The maximal spatial and orientational displacements were adjusted to obtain acceptance ratios of about 0.85 . As we fix the volume $V$ and the total particle number $N$, the mean density in the simulation box is prescribed, but not the bulk density. We determine this a posteriori from the (plateau) value of the density profile away from the wall, that is in the middle of the simulation box.

For the size ratio $L / \sigma=1.5$ we obtained reliable results for the density and order parameter profiles; those are presented below. Increasing the needle length (we checked $L / \sigma=5$ ) leads to significant slowing down of the equilibration of the system, and hence prohibited the obtaining of high-quality data.

\subsection{Order parameters and the wall sum rule}

In order to analyse the behaviour of the non-spherical particles we consider two characteristic distributions obtained from the full profile $\rho_{\mathrm{A}}(\mathbf{r}, \boldsymbol{\Omega})$. The first is the orientation averaged profile which describes the distribution of the centres of the spheres, regardless of their tail orientation, given by

$$
\bar{\rho}_{\mathrm{A}}(\mathbf{r})=\int \frac{\mathrm{d}^{2} \Omega}{4 \pi} \rho_{\mathrm{A}}(\mathbf{r}, \boldsymbol{\Omega}) .
$$

The second is an orientational order parameter

$$
\langle\cos \theta\rangle=\bar{\rho}_{\mathrm{A}}(\mathrm{r})^{-1} \int \frac{\mathrm{d}^{2} \Omega}{4 \pi} \rho_{\mathrm{A}}(\mathbf{r}, \boldsymbol{\Omega}) \cos \theta,
$$

that varies between -1 for particles with tails pointing away from the wall and +1 for particles with tails pointing towards the wall. It vanishes in the (isotropic) bulk fluid. As we restrict ourselves to the study of states with planar symmetry, $\bar{\rho}_{\mathrm{A}}$ and $\langle\cos \theta\rangle$ depend only on $z$.

For hard spheres, that is $L=0$, the density profiles against a hard wall satisfy the well-known sum rule $\beta P=\rho\left(R^{+}\right)$, where $\beta=1 / k_{\mathrm{B}} T, k_{\mathrm{B}}$ is the Boltzmann constant, $T$ is absolute temperature, $P$ is the bulk pressure and $R^{+}=(\sigma / 2)^{+}$is the distance of the closest approach between the sphere centre and the wall surface [19] (the upper plus indicates a limit from above). This sum rule was subsequently generalized to systems of hard anisotropic particles against a hard wall [20] which, for a one-component system of amphiphiles, is given by

$$
\beta P=\int \frac{\mathrm{d}^{2} \Omega}{4 \pi} \rho_{\mathrm{A}}\left(z^{+}(\theta), \theta\right),
$$

where $z^{+}(\theta)$ is the distance of the closest approach between the particle centre and the surface of the wall for a particle with orientation $\theta$, see equation (2).

\section{Results \\ 4.1. Density and order parameter profiles: comparison with simulations}

To assess the performance of the amphiphile functional we compare positional and orientational order parameter profiles (equations (3) and (4)) from DFT and MC simulation for $L / \sigma=1.5$. Owing to the complicated shape of the amphiphilic particles it is not obvious to what extent our approximate functional will capture the subtle packing effects which determine the interfacial structure in this system. Figure 2 shows the angle averaged density profiles $\bar{\rho}_{\mathrm{A}}(z)$. At low densities $\left(\eta_{\mathrm{A}}=\right.$ 0.05212 in figure $2(a)$ and $\eta_{\mathrm{A}}=0.10437$ in figure $2(b)$ ) the amphiphile tails strongly perturb the density profiles from those of pure hard spheres at the same packing fraction. At these low densities the contact value is reduced compared to pure hard spheres owing to wallamphiphile tail collisions which tend to move the sphere centres away from the wall. Note that the lowering of the contact value of the averaged density profile does not imply lowering of the bulk pressure (via relation through the wall sum rule), as collisions of the tails with the wall also need to be taken into account. We shall discuss this issue in the next subsection. The kink in the profile at $z=2 \sigma$ corresponds to a distance where an amphiphile with $\theta=0$ makes contact with the wall. For these low densities we find good agreement between simulation and theory.

As $\eta_{\mathrm{A}}$ is increased the packing of the amphiphile heads begins to dominate and the structure becomes closer to that of hard spheres $\left(\eta_{\mathrm{A}}=0.15585\right.$ in figure $2(c)$ and $\eta_{\mathrm{A}}=0.20728$ in figure $\left.2(d)\right)$. For high densities $\left(\eta_{\mathrm{A}}=0.25810\right.$ in figure $2(e)$ and $\eta_{\mathrm{A}}=0.30751$ in figure $2(f))$ the profile is very close to that of hard spheres and the presence of the amphiphile tails acts only as a weak perturbation. The effect of the tails is overwhelmed by the sphere packing.

Figure 3 shows the orientational order parameter profile $\langle\cos \theta\rangle$ for two typical densities, namely $\eta_{\mathrm{A}}=$ 0.05 and 0.25 . Despite the markedly differing values the 

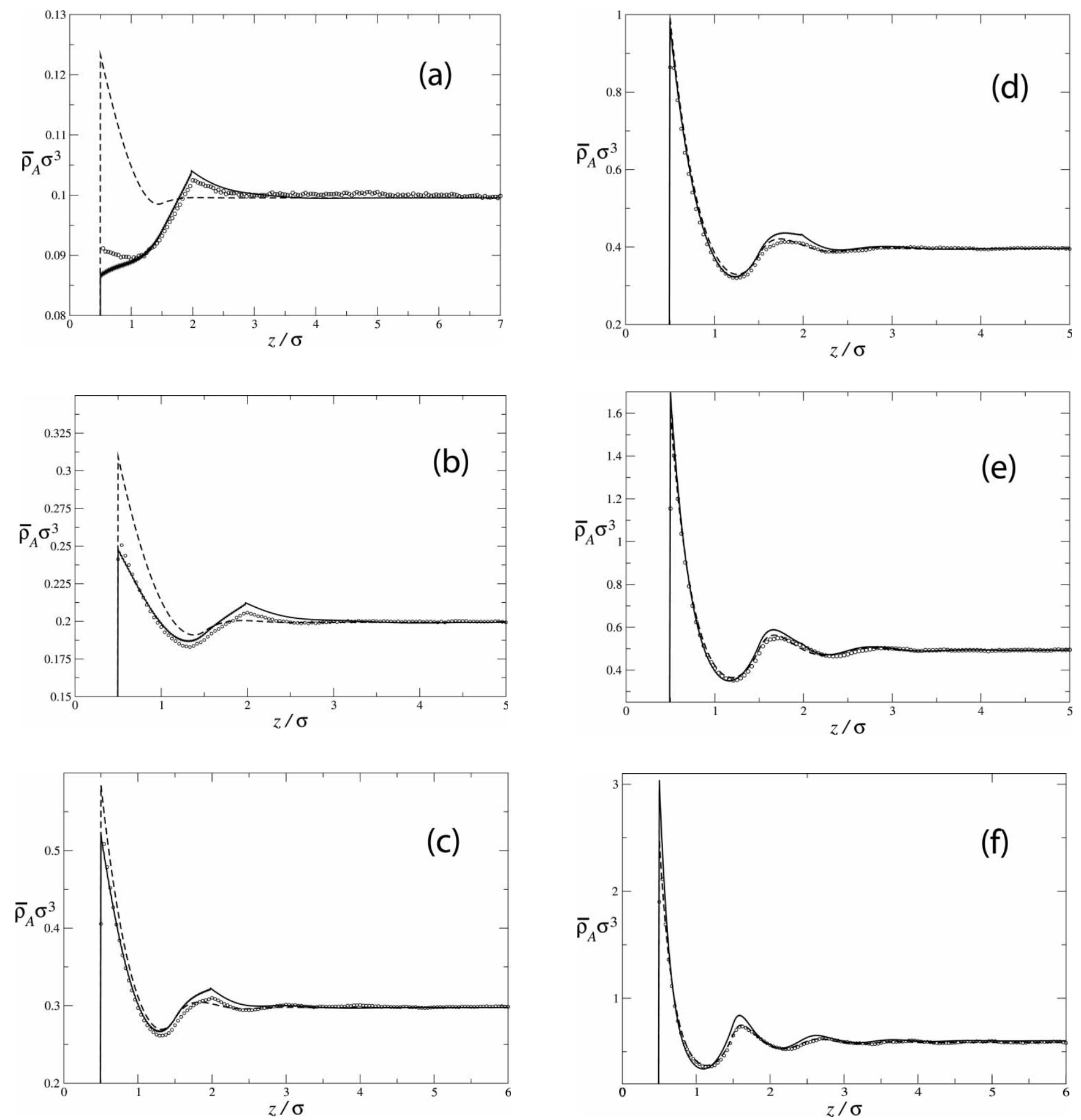

Figure 2. Angle averaged amphiphile density profiles $\bar{\rho}_{\mathrm{A}}$ as a function of the scaled distance from the wall $z / \sigma$ for $L / \sigma=1.5$ obtained from simulations (symbols) and DFT (solid curve). For comparison we also plot the DFT results for the pure hard sphere case $(L=0)$ for the same densities (dashed curve). Packing fractions are $(a) \eta_{\mathrm{A}}=0.05212 ;(b) \eta_{\mathrm{A}}=0.10437$; (c) $\eta_{\mathrm{A}}=0.15585 ;(d) \eta_{\mathrm{A}}=0.20728 ;(e) \eta_{\mathrm{A}}=0.25810 ;(f) \eta_{\mathrm{A}}=0.30751$.

simulation yields two very similar profiles, the only significant difference being the weak oscillations for $\eta_{\mathrm{A}}=0.25$ induced by sphere packing. The 'triangle' structure for $z<2 \sigma$ is almost completely determined by the reduction in solid angle available to each amphiphile tail when close to the wall; interactions between the amphiphiles appear to have little effect. In fact the profile for $\eta=0.05$ already lies very close to the low density limit with a small positive enhancement at $z=2 \sigma$. While the functional gives a good account of the 

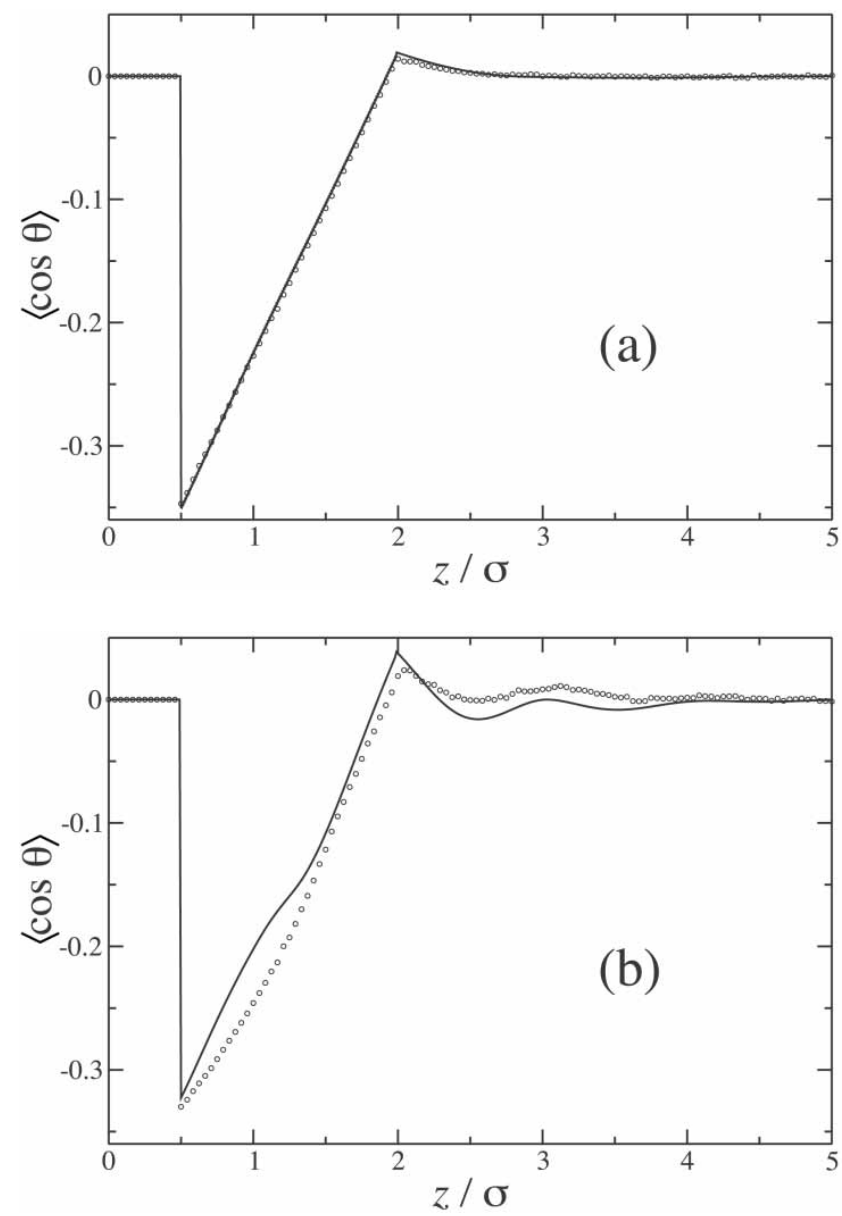

Figure 3. The orientational order parameter profiles $\langle\cos \theta\rangle$ as a function of the scaled distance $z / \sigma$ from the wall obtained from simulation (circles) and DFT (curves). Packing fractions are $(a) \eta_{\mathrm{A}}=0.05$ and $(b) \eta_{\mathrm{A}}=0.25$.

low density behaviour of $\langle\cos \theta\rangle$ when compared to simulation, for higher densities the agreement is poor. As $\eta$ is increased the simulation results show that (i) the contact value increases (becomes less negative), (ii) there is a deviation from linearity between contact and $z=2$, (iii) there is enhancement at $z / \sigma=2$ and, (iv) oscillations develop. All of these features are captured by the DFT, but are grossly overestimated. A possible explanation could lie in our assumption that there is no ordering parallel to the wall. This is implicit in our calculation of one-dimensional ( $z$-dependent) density profiles but is still accounted for within the simulation. While this presents an interesting possibility, the numerical minimization of the amphiphile functional [10] allowing for such ordering presents a formidable numerical challenge and is beyond the scope of the present work.

Preliminary simulation results for longer tails, $L / \sigma=5$, and $\eta_{\mathrm{A}}=0.2$ indicate deviations from the theoretical profiles. In particular we observe a dip in $\rho_{\mathrm{A}}(z)$ ranging from about $z=2 \sigma$ to $z=5 \sigma$, which is about $15 \%$ lower than the bulk density. This feature is absent in the starting configuration and develops over simulation time, albeit no equilibrated state could be reached. Our current DFT implementation is limited to planar symmetry, and does not reproduce this feature. Thus it is interesting to speculate that this could be an indication of lateral ordering at the wall, for example wall-induced micelle formation or freezing. We leave this issue for future work.

\subsection{Wall contact distribution}

The hard wall sum rule, equation (5), is an exact theorem. When used in an approximative DFT treatment it is expected, from general considerations, that the equality holds provided both sides of the equation are obtained from the same theory, that is the pressure (lefthand side of equation (5)) is obtained from the bulk equation of state while the right-hand side is obtained from the density profile which minimizes the functional. The sum rule should be satisfied by any non-local functional and thus provides a useful check of our numerical procedure. For each $\eta_{\mathrm{A}}$ value we find the sum rule (equation (5)) to be satisfied. The present study introduces two non-trivial complications over existing studies of hard particles at a hard wall: (i) the amphiphile particles are non-convex and (ii) the functional contains only an approximate Mayer function. To the best of our knowledge the sum rule has never been explicitly tested for either of these cases. Satisfaction of the sum rule for amphiphiles proves to be considerably more demanding than for pure hard spheres or the sphere-needle binary mixture [18]. A rather fine numerical mesh is required to achieve good accuracy; we use 200 spatial grid points per $\sigma$ in $z$ and 150 angular steps in the range $0 \leq \theta \leq \pi$. To provide good resolution in rapidly varying regions we employ a non-uniform grid in $\theta$.

In addition to providing a numerical check, the investigation of the sum rule also yields insight into the liquid structure at the wall. Using $\langle\cos \theta\rangle$ as a measure of orientational order does demonstrate the average tendency of the amphiphile tails to point away from the wall. However, much detail is washed out in the averaging process. Figure 4 shows the contact distribution $\rho_{\mathrm{A}}^{+}(\theta) \equiv \rho_{\mathrm{A}}\left(z^{+}(\theta), \theta\right) \sin \theta$ (the integrand of equation (5)), for several values of $\eta_{\mathrm{A}}$. For low $\eta_{\mathrm{A}}$ values the function is smooth (in $\theta$ ) but as $\eta_{\mathrm{A}}$ increases it becomes sharply peaked at the point labelled a. A careful numerical integration is thus required to evaluate the right hand side of equation (5). For the size ratio considered $(L / \sigma=1.5)$ the peak is located at an angle $\theta_{\mathrm{a}}=1.318$, identifying the most common geometrical arrangement of a particle at the wall. This a-type 


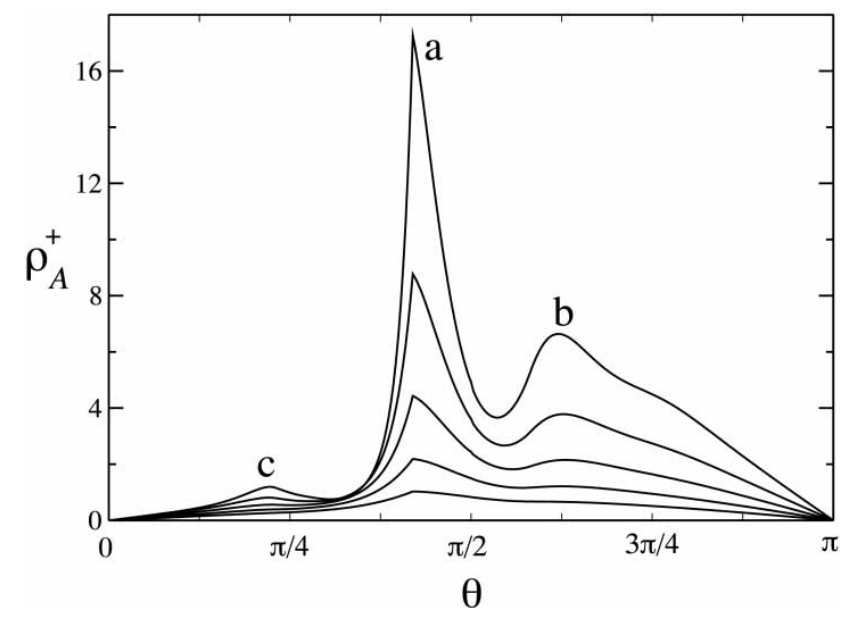

Figure 4. Amphiphile contact distribution $\rho_{\mathrm{A}}^{+} \equiv \rho_{\mathrm{A}}\left(z_{\mathrm{A}}^{+}(\theta), \theta\right)$ $\sin \theta$ for packing fraction $\eta_{\mathrm{A}}=0.15-0.35$ in intervals of 0.05 (from bottom to top). $\theta$ is the angle between the amphiphile tail and the wall surface normal. The features labelled a, b, c correspond to the configurations shown in figure 5 .

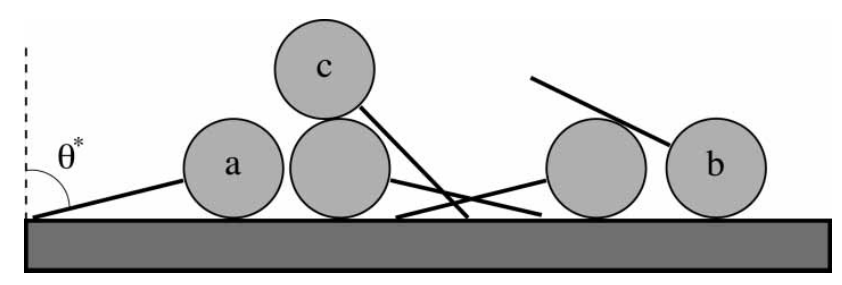

Figure 5. Typical configurations of model amphiphiles at contact with the hard wall, shown here for $L / \sigma=1.5$. The configurations labelled a, b, c correspond to the peaks in the contact distribution, see figure 4 .

configuration is shown in figure 5. It consists of an amphiphile with both the spherical surface and tail end simultaneously touching the wall. As $\eta_{\mathrm{A}}$ increases this configuration becomes more dominant and particles thus arranged play the largest role in balancing the bulk pressure via equation 5 . We have checked that for longer tail lengths $(L / \sigma=5)$ the peak develops at lower $\eta_{\mathrm{A}}$ values as it becomes easier to 'flatten' the amphiphiles against the wall. Hence in general we expect $\theta_{\mathrm{a}}=$ $\arccos [1 /(1+2 L / \sigma)]$.

For $\eta_{\mathrm{A}}>0.3$ two more peaks become apparent at angles $\mathrm{b}$ and $\mathrm{c}$ in figure 4 , each corresponding to a favoured amphiphile configuration at the wall. The interpretation of these higher order peaks is more subtle as they represent configurations involving pairs of amphiphile particles against the wall, see figure 5 . Configuration $b$ occurs when the amphiphile sphere surface touches the wall while the tail rests on top of a neighbouring sphere. If the needle touches the neighbouring surface such that it assumes the largest $\theta$ value possible for a given sphere-sphere separation, then for $L / \sigma=1.5,1.824<\theta_{\mathrm{b}}<2.094$ for sphere separations where the needle is tangent to the neighbouring sphere surface. This is in excellent agreement with the location of peak $b$ in figure 4. It should be noted that peak $a$ is sharp because amphiphiles with angles close to $\theta_{\mathrm{a}}$ all rapidly fall into a-type configurations; type-b configurations are more varied and include those where the needle has 'slid off' its neighbouring sphere to some extent. This accounts for the rounding of the peak. Also, the peak height of $b$ is lower than that of $a$ because $b$ configurations are unstable and can easily fall into type a. The peak $\mathrm{c}$ is small, even for $\eta_{\mathrm{A}}=0.35$, and is the most unstable of the three identified configurations. If the head of amphiphile $\mathrm{c}$ in figure 5 is positioned directly on top of the second amphiphile head, that is the line joining the sphere centres is normal to the wall and the tail touches the wall, an angle of $\theta_{c}=\arccos [3 /(1+2 L / \sigma)]$ is achieved. For $L / \sigma=1.5$ this yields $\theta_{\mathrm{c}}=0.723$, in excellent agreement with the peak location.

It is likely that the smooth background in the contact distribution (figure 4) also contains numerous other geometrically significant arrangements involving threeand higher-body structures but these are so weak that they cannot be resolved. The dominance of a-type configurations at large $\eta_{\mathrm{A}}$ values also provides an explanation for the increase in $\langle\cos \theta\rangle$ at contact with increasing $\eta_{\mathrm{A}}$ (see figure 3). As $\eta_{\mathrm{A}}$ is increased a-type configurations become more common, as $\theta_{\mathrm{a}}<\pi / 2$. Such configurations tend to make $\langle\cos \theta\rangle$ more positive.

\section{Conclusions}

In summary, we have investigated the structural correlations that arise near a hard wall in a hard body amphiphile fluid. In our simple model the amphiphile headgroup is taken to be a hard sphere and the tail is modelled as a rigid, vanishingly thin hard needle attached radially to the sphere. A fluid of such joined hard particles, when supplemented by species corresponding to water and oil (hard spheres and hard needles in this case) gives a simplistic representation of a ternary amphiphilic mixture. The simplicity stems from the absence of temperature as a relevant variable and hence the behaviour is solely governed by entropy.

Previous results for the fluid demixing phase behaviour indicated that the model indeed displays some of the features found in real systems [10]. Those results were obtained using a density functional approach that utilizes the fundamental measure concept to approximate the excess free energy. In this paper we presented the first application of the theory to an inhomogeneous situation. We chose a simple, albeit non-trivial test case, namely the pure amphiphile fluid against a hard wall, where non-trivial positional and orientational packing of particles is found. We have focused on the case of one-component amphiphiles in order to assess the 
accuracy of the DFT and have carried out Monte Carlo computer simulations to provide benchmark results.

We find good agreement between results from the theory and the simulations for the total (integrated over orientations) density, and reasonable agreement for the angular order parameter profile. We conclude that the theory gives a good account of the structural correlations that arise from the packing effects in this model. Owing to the particle shape, interesting behaviour at contact with the wall is found. The (orientation-dependent) density at contact with the wall displays a highly irregular, peaked structure, and we could qualitatively interpret the results in terms of typical particle configurations. In particular the most probable configuration is where head and tail touch the wall simultaneously.

As concerns future work, we mention the problem of how adding amphiphiles changes the free interface between demixed fluids in the sphere-needle mixture [18]. Furthermore whether the model leads to mesoscopic structures such as micelles is interesting. A particular challenge is to reveal the nature of the crystalline state(s).

We gratefully acknowledge inspiring discussions with D. W. Oxtoby, R. Evans, and H. Löwen. P. Bolhuis and D. Frenkel are thanked for pointing out reference [7] to us. The work of JMB was supported by the National Science Foundation (through grant CHE9800074 and the National Science Foundation Materials Research Science and Engineering Center at the University of Chicago).

\section{References}

[1] Somoza, A. M., Chacón, E., Mederos, L., and Tarazona, P., 1995, J. Phys.: condens. Matter, 7, 5753.

[2] Talanquer, V., and Охтовy, D. W., 2000, J. chem. Phys., 113, 7013.

[3] Napari, I., LaAksonen, A., and Strey, R., 2000, J. chem. Phys., 113, 4476.

[4] Napari, I., LaAksonen, A., and Strey, R., 2000, J. chem. Phys., 113, 4480.

[5] Duque, D., and Tarazona, P., 1997, J. chem. Phys., 107, 10207.

[6] Guerra, C., Somoza, A. M., and Telo da Gama, M. M., 1998, J. chem. Phys., 109, 1152.

[7] Bolnuis, P. G., and Frenkel, D., 1997, Physica A, 244, 45.

[8] van Duijneveldt, J. S., and Allen, M. P., Molec. Phys., 92, 855 .

[9] van Duijneveldt, J. S., Gil-Villeggas, A., Jackson, G., and Allen, M. P., 2000, J. chem. Phys., 112, 9092.

[10] Schmidt, M., and von Ferber, C., 2001, Phys. Rev. E, 64, 051115.

[11] Bolhuis, P., and Frenkel, D., 1994, J. chem. Phys., 101, 9869.

[12] Evans, R., 1979, Adv. Phys., 28, 143.

[13] Evans, R., 1992, Fundamentals of Inhomogeneous Fluids, edited by Henderson, D., (New York: Dekker), p. 85.

[14] Rosenfeld, Y., 1984, Phys. Rev. Lett., 63, 980.

[15] Rosenfeld, Y., 1994, Phys. Rev. E, 50, R3318.

[16] Rosenfeld, Y., 1995, Molec. Phys., 86, 637.

[17] Schmidt, M., 2001, Phys. Rev. E, 63, 050201(R).

[18] Brader, J. M., Esztermann, A., and Schmidt, M., 2002, Phys. Rev. E, 66, 031401.

[19] Henderson, J. R., and van Swol, F., 1985, Molec. Phys., 56, 1313.

[20] Holyst, R., 1989, Molec. Phys., 68, 391. 